\title{
Face Recognition and Hand Gesture Analysis System
}

\author{
Sunny Shahdadpuri ${ }^{1}$, Bhagwat Kakde ${ }^{2}$ \\ ${ }^{1}$ PG Research Scholar, Department of Electronics and Communication, R.K.D.F. Institute of Science \& Technology, Misrod, Bhopal, India. \\ ${ }^{2}$ Assistant Professor, Department of Electronics and Communication, R.K.D.F. Institute of Science \& Technology, Misrod, Bhopal, India
}

\begin{abstract}
An increasingly digital world, reliable personal authentication has become an important human Computer interface activity. National security, e-commerce and have access to a computer network. It is important to establish a person's identity, where some examples. Existing security measures depends on Based knowledge such as passwords, swipe cards or token -based approach and attitude to control access to physical and virtual spaces passport. Universal, such as methods, although very secure. Such as tokens, badges and access cards can be shared or stolen. Passwords and PIN Numbers can be stolen electronically. In addition, they cannot distinguish between authentic have access to or knowledge of the user and tokens. To make a system more secure and simple with the use of biometric authentication system such as face and hand gesture recognition for personal authentication. In this paper, a new Hidden Markov Model (HMM)-based face recognition system is proposed, having approximately $99 \%$ of recognition rate. With using quantized Singular Values Decomposition (SVD) coefficients as features describing blocks of face images and also hand gesture analysis system available using skin segmentation.
\end{abstract}

Keywords: Face Recognition, Hand Gesture Analysis System, HMM, SVD

\section{Introduction}

What is Authentication?

The process of identifying an individual usually based on a username and password. In security systems, authentication is distinct from authorization, which is the process of giving individuals access to system objects based on their identity. Authentication merely ensures that the individual is who he or she claims to be, but says nothing about the access rights of the individual.

Authentication is often discussed in terms of the three factors of authentication (i.e., three different kinds of things that can be used to authenticate an individual):

Something that is known to the individual (for example, a password, a personal identification number or PIN, an account number, favourite color, name of first pet);

Something that the individual has (for example, a bankcard, token, identity card, public-key digital certificate);

Something that the individual is (for example, a biometric, such as a facial image, retina scan or voice print) or does-a signature.

In some cases, any one of these factors can be used alone to authenticate an individual; in others, combinations are used. For example:

Access to a physically secure area using an identity card with an embedded chip (a smartcard) and a hand-scan biometric: This represents a two-factor authentication process: it relies on something the individual has (the smartcard) and something the individual is (the biometric).

Biometrics such as fingerprint, face and voice print offers means of reliable personal authentication that can address these problems and is gaining citizen and government acceptance.

\section{Biometric}

Biometrics is the science of verifying the identity of an individual through physiological measurements or behavioral traits. Since biometric identifiers are associated permanently with the user they are more reliable than token or knowledge based authentication methods. Biometrics offers several advantages over traditional security measures.The various biometric modalities can be broadly categorized as

- Physical biometrics: These involve some form of physical measurement and include modalities such as face, fingerprints, iris-scans, hand geometry etc.

- Behavioral biometrics: These are usually temporal in nature and involve measuring the way in which a user performs certain tasks. This includes modalities such as speech, signature, gait, keystroke dynamics etc.

- Chemical biometrics: This is still a nascent field and involves measuring chemical cues such as odor and the chemical composition of human perspiration.

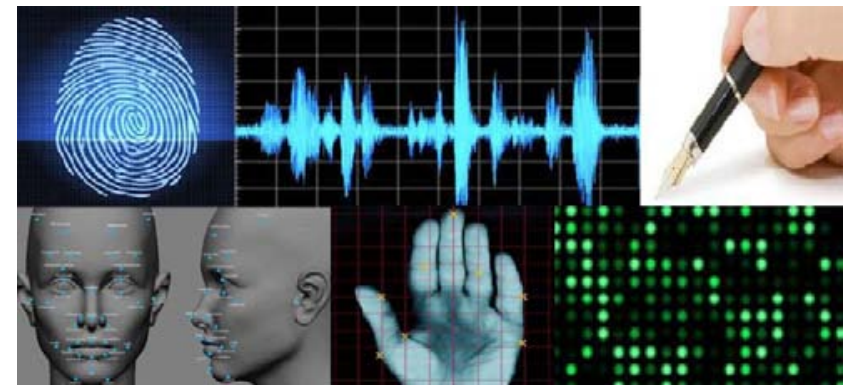

Figure 1: various biometric modalities: Fingerprints, speech, handwriting, face, hand geometry and chemical biometrics 


\section{Proposed Algorithm For Image Processing Based Authentication System}

Three stages of Authentication:

- Face detection

- Face recognition

- Hand gesture detection and recognition

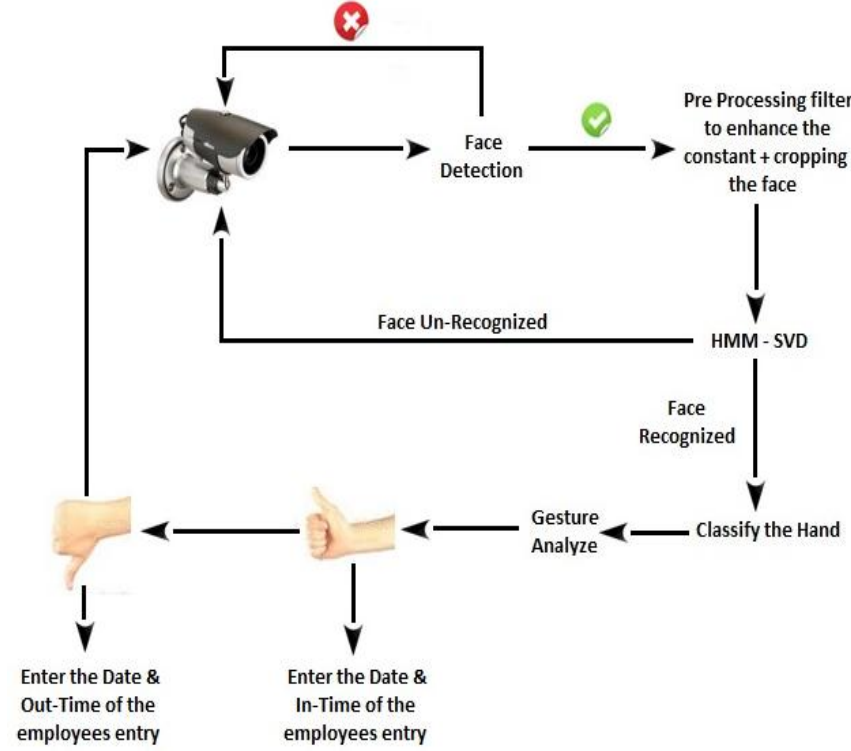

Figure 2: General block diagram of Image Processing based Authentication system

Project will contain few phases, phase one will be to detect the face, the camera will be set on video streaming once the employee is coming it takes a snapshot after detecting the face, in phase two which will be to extract the features necessary to recognize the face, we are thinking of using Gabor features, phase three will be making the right decision based on a data-base trained models, phase four will make the system say "Welcome to Work Mr...." and then wait for the employee hand gesture, after the gesture was make the system will analyze the hand gesture and enter the date time of the employee entrance.

\section{Face Detection and Face Recognition}

Face recognition is a biometric approach that employs automated methods to verify or recognize the identity of a living person based on his/her physiological characteristics. In general, a biometric identification system makes use of either physiological characteristics (such as a fingerprint, iris pattern, or face) or behavior patterns (such as hand-writing, voice, or key-stroke pattern) to identify a person. Because of human inherent protectiveness of his/her eyes, some people are reluctant to use eye identification systems. Face recognition has the benefit of being a passive, non intrusive system to verify personal identity in a —mural" and friendly way.

In general, biometric devices can be explained with a three step procedure (1) a sensor takes an observation. The type of sensor and its observation depend on the type of biometric devices used. This observation gives us a Biometric Signature" of the individual. (2) a computer algorithm -armalizes" the biometric signature so that it is in the same format (size, resolution, view, etc.) as the signatures on the system's database. The normalization of the biometric signature gives us a Normalized Signature" of the individual. (3) a matcher compares the normalized signature with the set (or sub-set) of normalized signatures on the system's database and provides a -similarity score" that compares the individual's normalized signature with each signature in the database set (or sub-set).

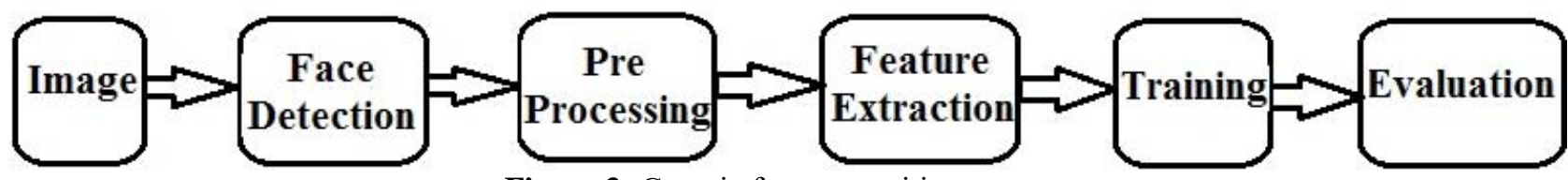

Figure 3: Generic face recognition systems

Face recognition starts with the detection of face patterns in sometimes cluttered scenes, proceeds by normalizing the face images to account for geometrical and illumination changes, possibly using information about the location and appearance of facial landmarks, identifies the faces using appropriate classification algorithms, and post processes the results using model-based schemes and logistic feedback system.

\section{Face Detection and Cropping Block}

This is the first stage of any face recognition system and the key difference between a semi-automatic and a fully automatic face recognizer. In order to make the recognition system fully automatic, the detection and extraction of faces from an image should also be automatic. Face detection also represents a very important step before face recognition, because the accuracy of the recognition process is a direct function of the accuracy of the detection process.

\section{Pre-Processing Block}

The face image can be treated with a series of pre-processing techniques to minimize the effect of factors that can adversely influence the face recognition algorithm. The most critical of these are facial pose and illumination.

\section{Feature Extraction Block}

In this step the features used in the recognition phase are computed. These features vary depending on the automatic face recognition system used. For example, the first and most simplistic features used in face recognition were the geometrical relations and distances between important points in a face, and the recognition algorithm' matched these distances the most widely used features in face recognition are KL or eigen faces, and the standard recognition algorithm' uses either the Euclidian or Mahalanobis distance to match features. 


\section{International Journal of Science and Research (IJSR) \\ ISSN (Online): 2319-7064}

Index Copernicus Value (2013): 6.14 | Impact Factor (2014): 5.611

\section{Face Recognition Block}

This consists of 2 separate stages: a training process, where the algorithm is fed samples of the subjects to be learned and a distinct model for each subject is determined; and an evaluation process where a model of a newly acquired test subject is compared against all existing models in the database and the most closely corresponding model is determined. If these are sufficiently close a recognition event is triggered.

Table 1: Comparative result of Different Methods

\begin{tabular}{|c|c|}
\hline Method (Learning algorithm) & Percentage of Error (\%) \\
\hline Pseudo 2D HMM + Gray tone & $5 \%$ \\
\hline Sliding HMM + Grey tone & $13 \%$ \\
\hline PDNN & $5 \%$ \\
\hline Eigenface & $10 \%$ \\
\hline Gabor filter + rank & $9 \%$ \\
\hline EBGM & $<20 \%$ \\
\hline Continuous n-tuple classifier & $3 \%$ \\
\hline Up-Down HMM + DCT & $16 \%$ \\
\hline Markov Random Fields & $13 \%$ \\
\hline SVM+PCA & $3 \%$ \\
\hline 1D HMM+SVD & $1 \%$ \\
\hline
\end{tabular}

As Table 1 shows the proposed system has a recognition rate of $99 \%$, a high rate that can simply be approximated to $100 \%$. The significance of this result is that such a high recognition rate is achieved using $\mathrm{HMM}+\mathrm{SVD}$ parameter. [6]

\section{HMM (Hidden Markov Model)}

The Hidden Markov Models are stochastic models which provide a high level of flexibility for modeling the structure of an observation sequence. They allow for recovering the (hidden) structure of a sequence of observations by pairing each observation with a (hidden) state. Hidden Markov Models (HMMs) represent a most famous statistical pattern recognition technique and can be considered as the state-of the- art in speech recognition. This is due to their excellent time warping capabilities, their effective self organizing learning capabilities and their ability to perform recognition and segmentation in one single step. They are used not only for speech and handwriting recognition but they are involved in modeling and processing images too. This is the case of their use in the face recognition field.

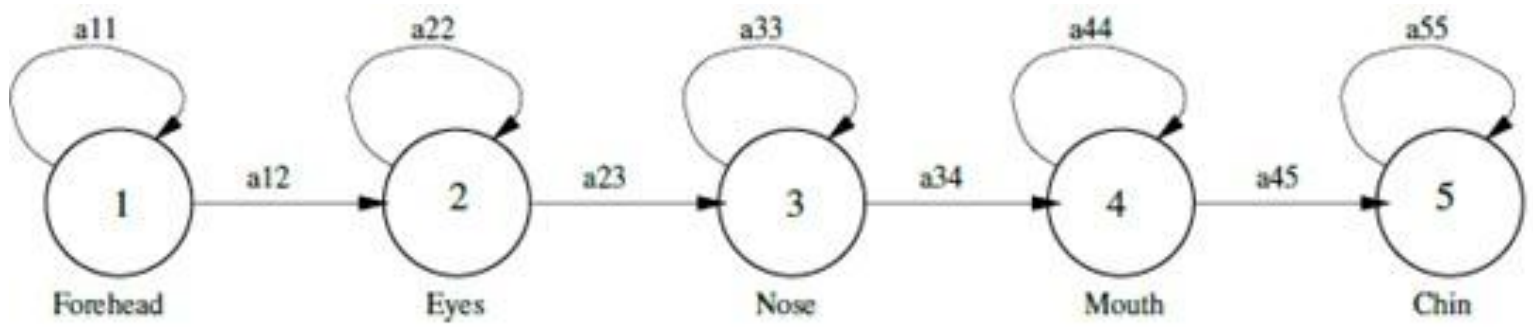

Figure 4: A one dimensional HMM for face recognition

Hidden Markov Models have been successfully used for speech recognition where data is essentially one dimensional. Extension to a fully connected two dimensional HMM has been shown to be computationally very complex. For frontal face images, the significant facial regions (hair, forehead, eyes, nose, and mouth) come in a natural order from top to bottom, even if the images are taken under small rotations in the image plane and/or rotations in the plane perpendicular to the image plane. Each of these facial regions is assigned to a state in a left to right $1 \mathrm{D}$ continuous HMM.

\section{Training and Recognition Algorithm}

\section{Feature extraction:}

In the context of face identification problem, we need to make a few image pre-processing; we need transform the face image to a uniform by level and size normalized. Then we need do fractal coding, we change face image in to matrix of fractal code. We looked upon each row of the fractal matrix as a vector. Then take these vectors clustering analysis with $\mathrm{K}$-means algorithm.

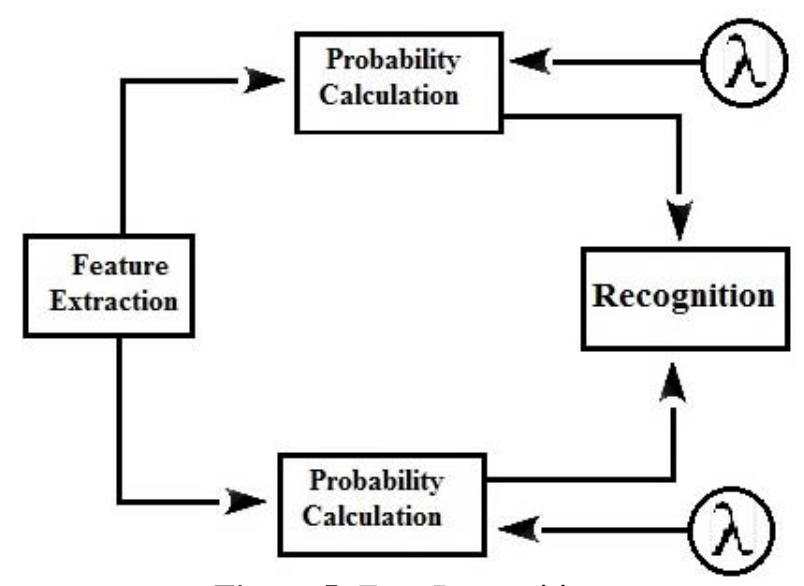

Figure 5: Face Recognition

\section{Training HMM:}

Train HMM by use of fact and record each HMM and cluster centres. This training process is conducted using the Baum-Welch algorithm as the detail image norms are real values, a continuous observation HMM is employed. Repeat it until all training-images are trained.

\section{Recognition algorithms:}

Feature extraction: A transform the face image to a uniform by level and size normalized. Then transform the face image to fractal matrix, use the matrix of fractal coding into HMM 


\section{International Journal of Science and Research (IJSR) \\ ISSN (Online): 2319-7064}

Index Copernicus Value (2013): 6.14 | Impact Factor (2014): 5.611

to calculate the probability. Choose the max matching probability recognition. If all of the matching probability is minor we consider as the face image can't find the matching face in this face database.

\section{SVD (Singular Value Decomposition) Parameter}

The Singular Value Decomposition (SVD) has been an important tool in signal processing and statistical data analysis. Singular values of given data matrix contain information about the noise level, the energy, the rank of the matrix, etc. As singular vectors of a matrix are the span bases of the matrix, and orthonormal, they can exhibit some features of the patterns embedded in the signal. SVD provides a new way for extracting algebraic features from an image. A SVD of a $m \times n$ matrix $X$ is any function of the form:

$\mathrm{X}=\mathrm{UWV}^{\mathrm{T}}(1)$

Where $\mathrm{U}(\mathrm{m} \times \mathrm{m})$ and $\mathrm{V}(\mathrm{m} \times \mathrm{m})$ are orthogonal matrix, and $\mathrm{W}$ is and $m \times n$ diagonal matrix of singular values with components $\sigma_{\mathrm{ij}=0,} \mathrm{i} \neq \mathrm{j}$ and $\sigma_{\mathrm{ii}}>0$. Furthermore, it can be shown that there exist non-unique matrices $\mathrm{U}$ and $\mathrm{V}$ such that $\sigma_{1} \geq \sigma_{2} \ldots \geq 0$. The columns of the orthogonal matrices $U$ and $\mathrm{V}$ are called the left and right singular vectors respectively; an important property of $\mathrm{U}$ and $\mathrm{V}$ is that they are mutually orthogonal. The main theoretical property of SVD relevant to face image recognition is its stability on face image. Singular values represent algebraic properties of an image. So because of these reasons and some experimental results, we find out that SVD is a robust feature extraction technique for face images.

Table 2: Comparative recognition results of some of the other methods.

\begin{tabular}{|c|c|c|c|}
\hline Method & $\begin{array}{c}\% \\
\text { Recognition }\end{array}$ & $\begin{array}{c}\text { Training } \\
\text { Time per } \\
\text { image }\end{array}$ & $\begin{array}{c}\text { Recognition } \\
\text { Time per } \\
\text { image }\end{array}$ \\
\hline PDBNN & 96 & $20 \mathrm{~min}$ & $\leq 0.1 \mathrm{sec}$. \\
\hline n-tuple & 86 & $0.90 \mathrm{sec}$. & $0.025 \mathrm{sec}$. \\
\hline 1DHMM + Wavelet & 100 & $1.13 \mathrm{sec}$. & $0.3 \mathrm{sec}$. \\
\hline Pseudo-2D HMM & 95 & N/A & $240 \mathrm{sec}$. \\
\hline DCT-HMM & 99.5 & $23.5 \mathrm{sec}$. & $3.5 \mathrm{sec}$. \\
\hline 1D HMM +SVD & 99 & $0.63 \mathrm{sec}$. & $0.28 \mathrm{sec}$. \\
\hline
\end{tabular}

Table 2 shows a comparison of the different face recognition techniques. As we can see our system has a recognition rate of $99 \%$ and a low computational cost. Besides these advantages, the system has low memory space consumption because of resizing the face images.

The recognition system works as the algorithm described figure 6 .

\section{Hand Gesture Recognition}

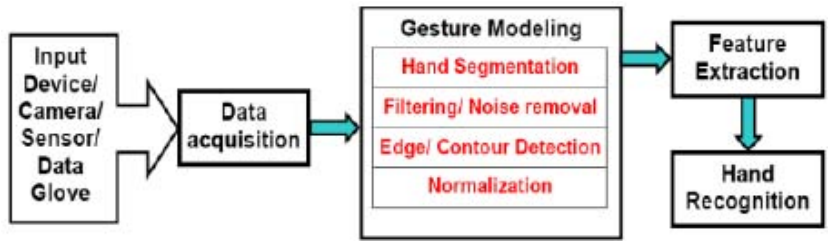

Figure 6: Generalized architecture of hand gesture recognition system

\section{Data Acquisition:}

For efficient hand gesture recognition, data acquisition should be as much perfect as possible. Suitable input device should be selected for the data acquisition. There are a number of input devices for data acquisition. Some of them are data gloves, marker, hand images (from webcam/ stereo camera/ Kinect 3D sensor) and drawings. Data gloves are the devices for perfect data input with high accuracy and high speed. It can provide accurate data of joint angle, rotation, location etc. for application in different virtual reality environments. At present, wireless data gloves are available commercially so as to remove the hindrance due to the cable. Coloured markers attached to the human skin are also used as input technique and hand localization is done by the colour localization. Input can also be fed to the system without any external costly hardware, except a low-cost web camera. Bare hand (either single or double) is used to generate the hand gesture and the camera captures the data easily and naturally (without any contact). Sometimes drawing models are used to input commands to the system. The latest addition to this list is Microsoft Kinect 3D depth sensor. Kinect is a 3D motion sensing input device widely used for gaming. It consists of a laser projector and a CMOS sensor for operation in any lighting conditions.

\section{Gesture Modeling:}

It is the next step after data acquisition and the success of the gesture recognition mostly depends on this stage. Different data received through the input devices are to be modeled properly depending up on the type of applications. Gesture modeling has four different steps, viz. hand segmentation, filter/ noise removal, edge/ contour detection and lastly normalization.

\section{Feature Extraction:}

Features are the crucial elements for hand gesture recognition. Large number of features, such as, shape, orientation, textures, contour, motion, distance, centre of gravity etc. can be used for hand gesture recognition. Hand gesture can be recognized using geometric features, like, hand contour, fingertips, finger detections. But these features may neither be always available nor reliable due to occlusions and illuminations. Some non-geometric features (such as colour, silhouette and texture) are also available for recognition. But they are inadequate for the purpose. Therefore, the image or the processed image can be fed to the recognizer to select the features automatically and implicitly, rather than using single type of feature alone. Following three approaches are useful for extraction of features.

\section{Hand Gesture Recognition:}

Once the appropriate features as mentioned above, have been extracted from the images and a suitable data set have been selected, the gestures can be recognized using standard machine learning techniques or a special-purpose classifiers. Several methods have been used for gesture recognition: template matching, dictionary look-up, statistical matching, linguistic matching, neural network and ad hoc method. 


\section{International Journal of Science and Research (IJSR) \\ ISSN (Online): 2319-7064}

Index Copernicus Value (2013): 6.14 | Impact Factor (2014): 5.611

\section{Flow of System}

Face Detection and Recognition:

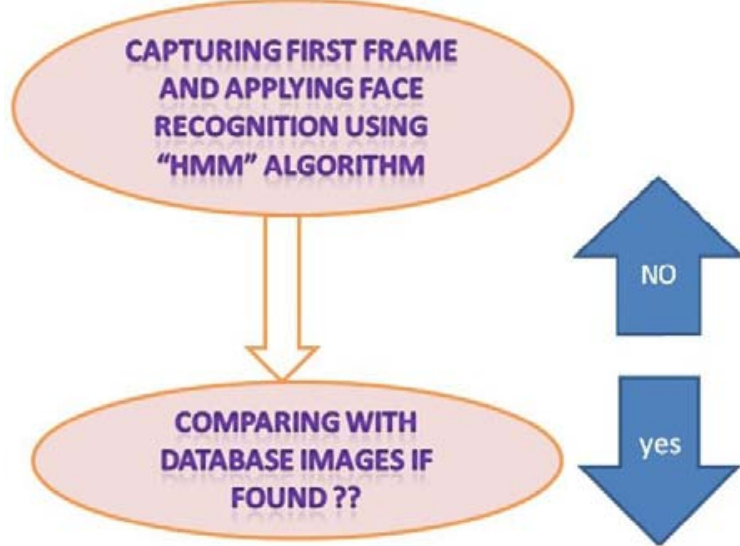

Figure 7: Face Detection and Recognition Process

\section{Hand Gesture Detection:}

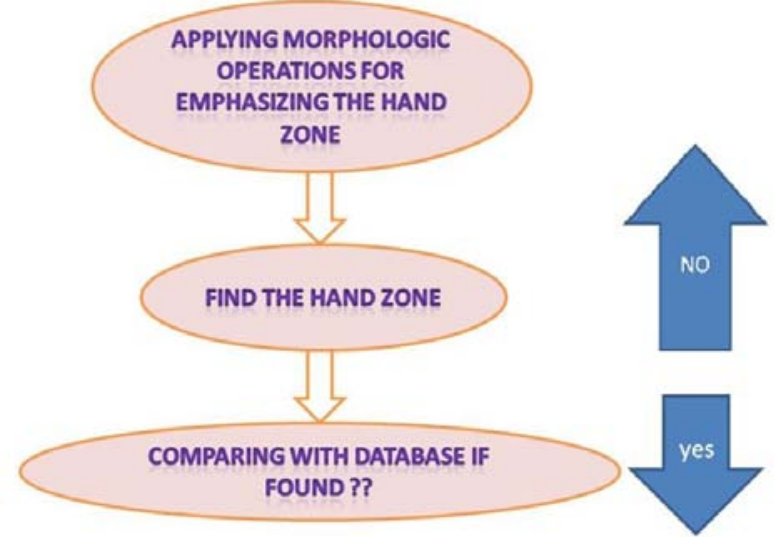

Figure 8: Hand Gesture Detection

Time and Date Record:

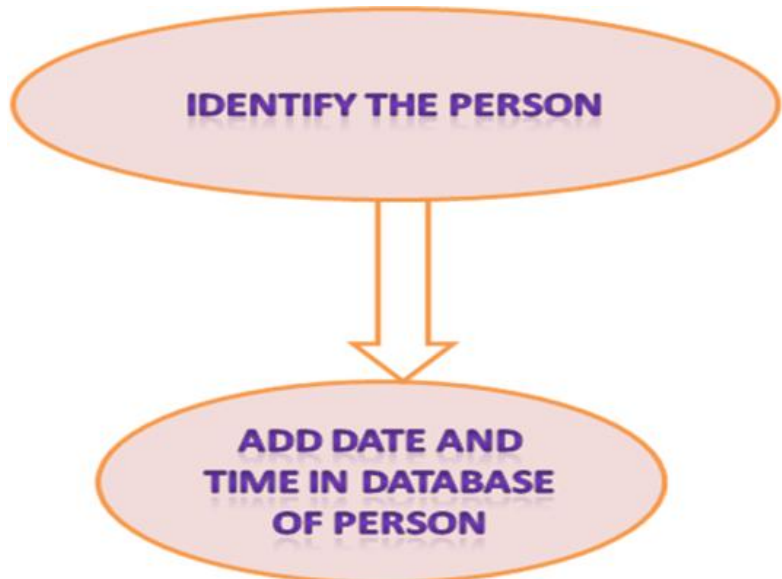

Figure 9: Times and Date Record

\section{Simulation Result in MATLAB}

Stage 1: Run code in mat-lab file. This code is made by using HMM+SVD algorithm.

Stage 2: After running the code it will show one box which ask to train face of employee.
Here for face detection we use Viloa Jones algorithm. It detects only faces. It will create frame towards face. It only detects face, not recognize. Here different types of algorithms are available but here I have used HMM+SVD technique because in this error ratio is only $1 \%$ comparing to other system. And rate of recognition is about to $99 \%$. Here it works in two phase,

a) Training

b) Face Recognition

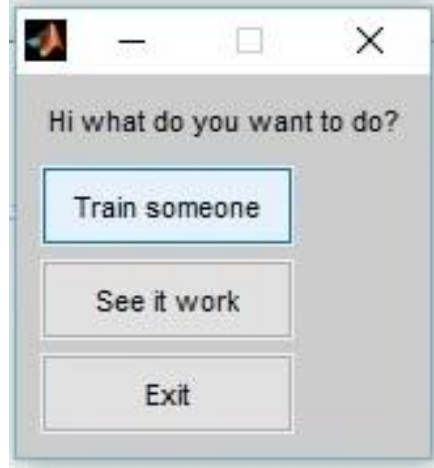

Figure 9: Train Person

Stage 3: Enter the employee's name.

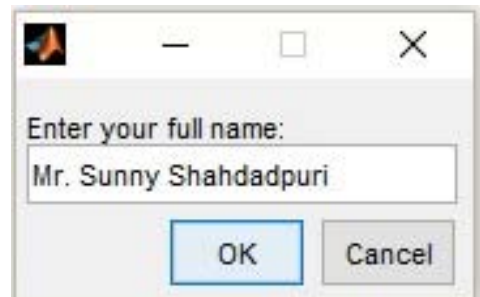

Figure 10: Write Person's Name

Stage 4: Now it will take up to 230 photographs of different angles of face in 5 seconds. And ask for train the photographs.

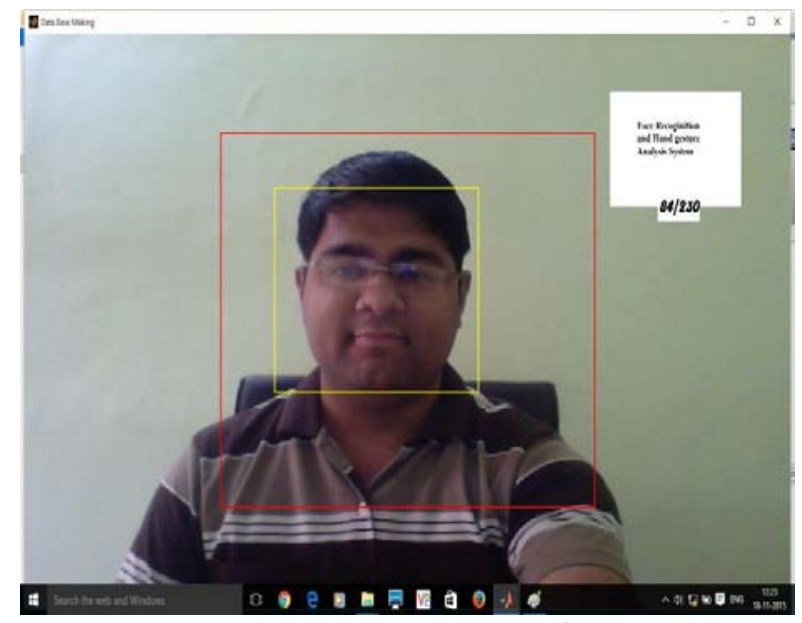

Figure 11: Image Capturing

Stage 5: For first time training the 230 photographs it will take around 10 to 15 minutes. As soon as training of photographs was completed, data base is ready of that employee. And it will show message of training were done using SVD parameter. It will save all data in .mat (database file) file. 


\section{International Journal of Science and Research (IJSR) \\ ISSN (Online): 2319-7064}

Index Copernicus Value (2013): 6.14 | Impact Factor (2014): 5.611

\begin{tabular}{l}
\hline- \\
Train the database now? \\
Yes \\
No \\
\hline
\end{tabular}

Figure 11: Train Person`s Face Image Database

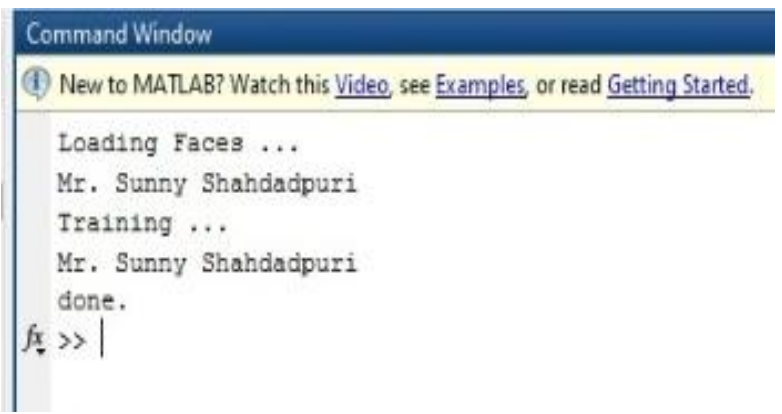

Figure 12: Training Face Images in MATLAB

Stage 6: Now system is ready for the work. It will ask - se the work"

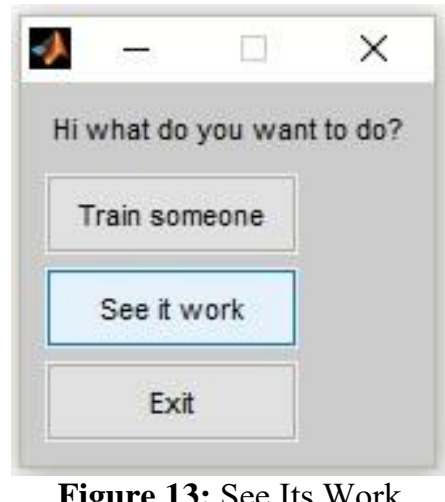

Stage 7: When person will come in default area, camera automatically captures the image of person. If person is outside of default area or in between that it will show No Person Found".

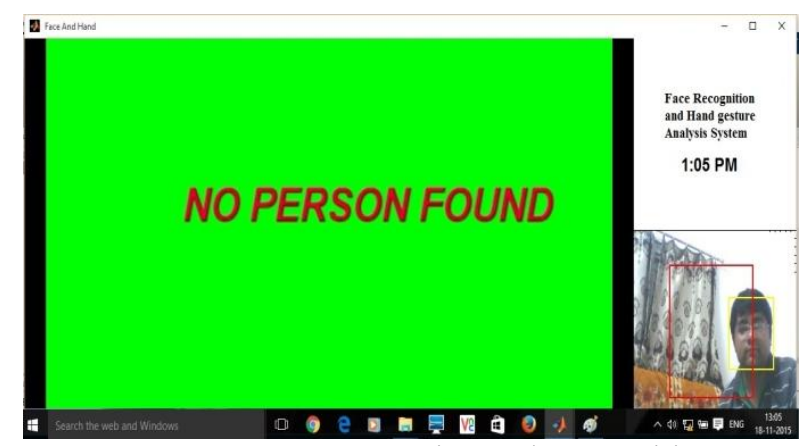

Figure 14: Face Detection and Recognition

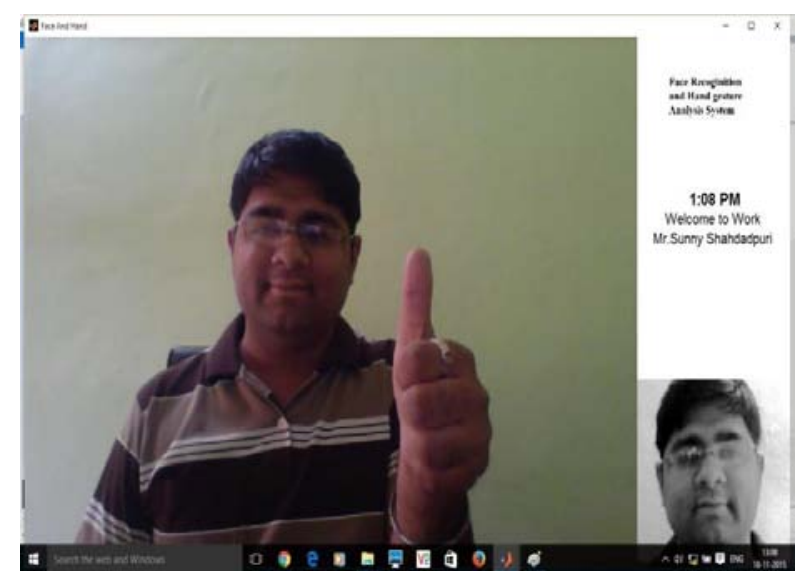

Figure 15: Person Recognized at IN Time with Up Hand Gesture

Stage 8: Here HMM recognize the face of employee \& SVD parameter calculate the 5 spot of face using database .mat file and check that image of that person is available in database or not. It will take only 0.28 second. If calculation is perfect and matching with employee image it shows the message on LCD -Welcome to Work Mr..." with this I will rise my hand with sign Thumbs Up so it HMM \& SVD recognize and calculate parameter and automatically one excels sheet will generated with date and entry time.

Stage 9: Now at the end of day when employee leaves from office once again whole process is repeated and message will comes on LCD screen -GoHome. Drive safely" and again by showing the Thumbs down sign it will automatically update that excels sheet with leave time.

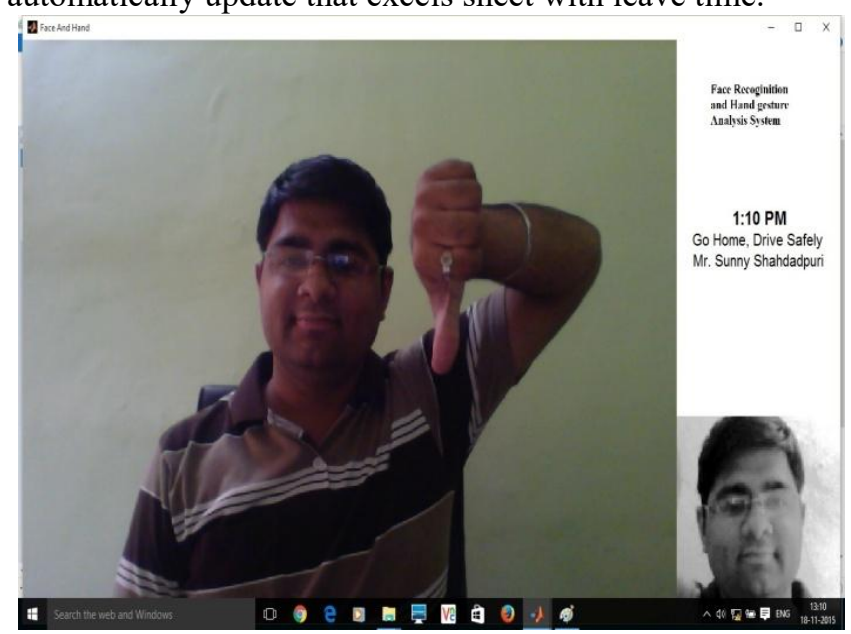

Figure 16: Person Recognized at OUT Time with Down Hand Gesture 


\section{International Journal of Science and Research (IJSR) \\ ISSN (Online): 2319-7064}

Index Copernicus Value (2013): 6.14 | Impact Factor (2014): 5.611

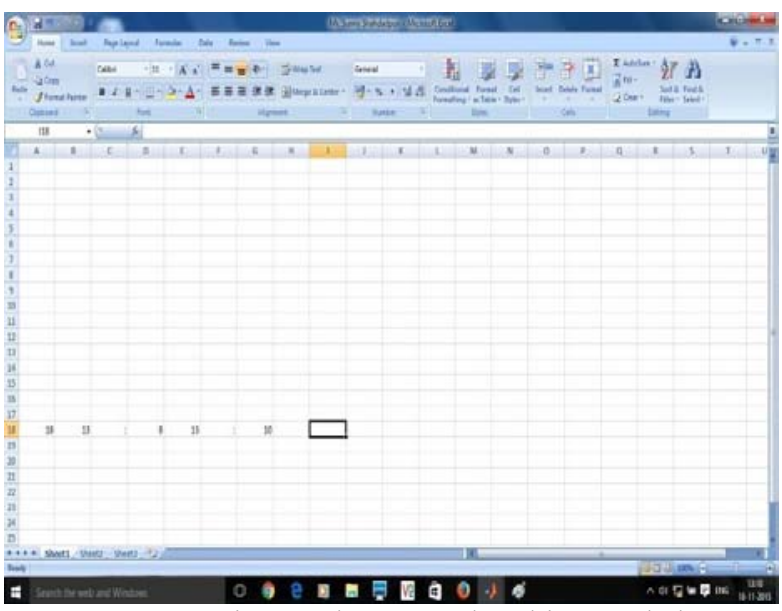

Figure 17: Time and Date updated in excel sheet

Stage 10: If another person comes in that recognition area who is not employee of the company and whose database is not available in .mat file it will automatically shows message No Person Found" message on LCD screen.

\section{Conclusions}

In the above system, we discussed about to make a system more secure and simple with the use of biometric authentication with the help of HMM algorithm with SVD parameters and also used skin segmentation, morphological operators are used for recognition system. In this system, approximately having a recognition rate of $99 \%$, the system was very fast.

This system could be useful in Enterprise and E-Government services, National Security, Airport, Bank, Offices, colleges, Biometric Time Attendance System.

\section{References}

[1] H. Miar-Naimi and P. Davari "A New Fast and Efficient HMM-Based Face Recognition System Using 7-State HMM Along With SVD Coefficients", Iranian Journal of Electrical \& Electronic Engineering, Vol. 4, Nos. 461 \& 2, Jan. 2008

[2] Rowayda A. Sadek "SVD Based Image Processing Applications: State of The Art, Contributions and Research Challenges", ,(IJACSA) International Journal of Advanced Computer Science and Applications, Vol. 3, No. 7, 2012

[3] Mukundhan Srinivasan, Nivas Ravichandran "A New Technique For Face Recognition Using 2D-Gabor Wavelet Transform With 2D-Hidden Markov Model Approach”, 978-1-4673-4862-1/13/\$31.00 (C2013 IEEE

[4] Mukundhan Srinivasan and Sriram Raghu "Comparative Study on Hidden Markov Model versus Support Vector Machine A Component-Based Method for Better Face Recognition", 2013 UKSim 15th International Conference on Computer Modelling and Simulation.

[5] Arpita Ray Sarkar G. Sanyal S. Majumder "Hand Gesture Recognition Systems: A Survey”, , International Journal of Computer Applications (0975 - 8887) Volume 71-No.15, May 2013
[6] S. Tolba, A.H. El-Baz, and A.A. El-Harby "Face Recognition: A Literature Review”, International Journal of Signal Processing 2;2 2006

\section{Author Profile}

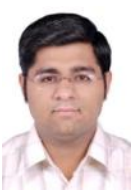

Mr. Sunny Shahdadpuri is PG Research Scholar, Department of Electronics and Communication, R.K.D.F. Institute of Science \& Technology, Misrod, Bhopal, India

Mr. Bhagwat Kakde, Assistant Professor, Department of Electronics and Communication, R.K.D.F. Institute of Science \& Technology, Misrod, Bhopal, India. 